\title{
Des bibliothèques ecclésiastiques aux bibliothèques publiques
}

l'exemple des vosges, 1789-1840

From Ecclesiastical Libraries to Public Libraries. The Example of the Vosges, 1789-1840

Jean Paul Rothiot

\section{(2) OpenEdition}

\section{Journals}

Édition électronique

URL : https://journals.openedition.org/ahrf/12210

DOI : $10.4000 /$ ahrf.12210

ISSN : 1952-403X

Éditeur :

Armand Colin, Société des études robespierristes

Édition imprimée

Date de publication : 1 décembre 2011

Pagination : 29-52

ISSN : 0003-4436

\section{Référence électronique}

Jean Paul Rothiot, «Des bibliothèques ecclésiastiques aux bibliothèques publiques », Annales historiques de la Révolution française [En ligne], 366 | octobre-décembre 2011, mis en ligne le 01 décembre 2014, consulté le 01 juillet 2021. URL : http://journals.openedition.org/ahrf/12210 ; DOI : https://doi.org/10.4000/ahrf.12210 


\title{
DES BIBLIOTHÈQUES ECCLÉSIASTIQUES AUX BIBLIOTHËQUES PUBLIQUES L'EXEMPLE DES VOSGES, 1789-1840
}

\begin{abstract}
Les bibliothèques publiques du département des Vosges, Épinal, Mirecourt, Neufchâteau, Remiremont et Saint-Dié, possèdent actuellement des fonds anciens constitués à partir des ouvrages provenant des riches abbayes vosgiennes. Plusieurs milliers d'ouvrages sont ainsi précieusement conservés; à Épinal, ils se trouvent dans les bois de la bibliothèque, datant du xviii ${ }^{e}$ siècle et provenant des bénédictins de Moyenmoutier. À ces imprimés s'ajoutent manuscrits et incunables. Cet article se propose d'étudier le processus de passage de ces livres des monastères aux bibliothèques publiques.
\end{abstract}

Mots-clés : Révolution, bibliothèques, biens nationaux, Vosges, abbaye, émigré

La vente des biens du clergé comme biens nationaux s'accompagne de la saisie des bibliothèques et des archives des institutions ecclésiastiques. "Cette saisie constitue un moment important de la constitution de notre patrimoine écrit $»^{1}$, en effet les livres confisqués ne sont pas vendus, car leur valeur culturelle a été reconnue par les comités de la Constituante, des experts doivent dresser la liste des ouvrages de chaque bibliothèque et en faire rapport à l'Assemblée. En 1791, le comité d'aliénation évalue à 4194412 le nombre de volumes déjà saisis dans les 4500 maisons religieuses de la France 2 . Dans les Vosges, 41000 volumes sont ainsi répertoriés et les bibliothèques les plus riches, Moyenmoutier, Senones,

(1) Bernard Bodinier, L'événement le plus important de la Révolution, la vente des biens nationaux, Paris, Société des études robespierristes, CTHS, 2000, p. 412-414.

(2) Dominique VArry, Histoire des bibliothèques françaises, Les Bibliothèques de la Révolution et du XIX siècle : 1789-1914, Paris, Promodis, Éditions du Cercle de la Librairie, 1991. 
Étival ou Mureau avec plusieurs milliers d'ouvrages chacune, font bonne figure à côté d'autres bibliothèques provinciales, même si elles sont loin des parisiennes (60120 à Sainte-Geneviève, 560459 à Saint-Germaindes-Prés... $)^{3}$.

Fig. 1 : La bibliothèque bénédictine de Moyenmoutier, XVIII siècle, au cour de la BMI d'Épinal. Cliché JPR.

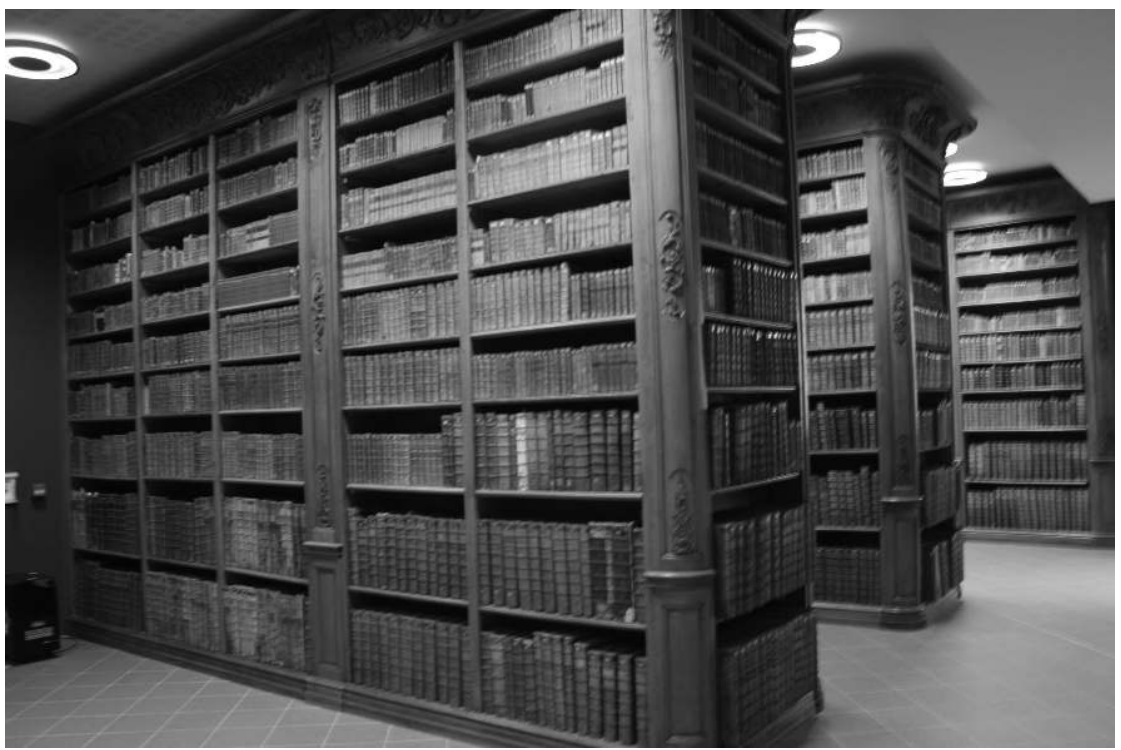

Si les lois, décrets et instructions émanant des assemblées nationales successives et des commissions chargées des bibliothèques sont bien connus ${ }^{4}$, leur mise en application dans les départements reste à étudier après le travail de Dominique Varry sur l'Eure ${ }^{5}$. Comment dans les Vosges, les administrations du département et des districts mettent-elles en application ces législations? Comment les inventaires sont-ils effectués et quelle est la destination des livres : sont-ils restés sur place, sontils rassemblés au chef-lieu de district, ou à celui du département? Les rapports de force et l'évolution des politiques nationales ont influé sur la destinée des livres. Enfin, se pose la question de la «dilapidation»,

(3) Bernard Bodinier, op. cit., p. 412.

(4) Dominique VARrY, Histoire des bibliothèques françaises... op. cit.

(5) Id., "Sous la main de la Nation ». Les bibliothèques de l'Eure confisquées sous la Révolution française, Centre national d'étude du XVIII ${ }^{\mathrm{e}}$ siècle, Ferney-Voltaire, 2005. 
du «vandalisme révolutionnaire »: sur les 41000 ouvrages recensés en 1791, il n'en reste aujourd'hui qu'un quart. Par quel processus ces dizaines de milliers d'ouvrages ont-ils disparu, à quelles époques, sous quels régimes? Est-ce le résultat du « vandalisme révolutionnaire »? Les inventaires des biens mobiliers ecclésiastiques, ceux des bibliothèques ecclésiastiques, les correspondances entre les administrations, quelques sources des fonds anciens des bibliothèques municipales, ont été utilisés ${ }^{6}$. Nous donnerons une vue globale sur le département et nous préciserons avec un exemple local, celui de Mirecourt, un des chefs-lieux de district, dont les archives sont particulièrement abondantes.

Tout d'abord, les biens ecclésiastiques ont été « mis dans la main de la nation » et les premiers inventaires sont réalisés en 1790-1791; ensuite les bibliothèques des couvents sont regroupées et des bibliothèques «nationales » de district constituées de 1791 à l'an III pour préserver ces biens essentiels à la culture, mais avec quelle efficacité? Enfin, les transformations intervenues sous le Directoire et le Consulat montrent une volonté de rationalisation, qui se veut en réaction avec la période précédente.

\section{Mise à la disposition de la nation et inventaires}

La mise à la disposition de la nation des biens ecclésiastiques s'accompagne d'états sommaires, qui sont, sous l'influence des comités de l'Assemblée nationale, repris pour des inventaires plus complets car l'intérêt des bibliothèques ecclésiastiques est reconnu et le gigantesque travail national d'inventaire doit, à terme, mener à la constitution d'un catalogue général national ${ }^{7}$. Cette deuxième vague d'inventaires est menée assez rapidement dans les Vosges, puisque le relevé des bibliothèques des 83 départements, achevé le 23 février 1791, indique 41009 ouvrages imprimés recensés dans les Vosges, loin derrière la Meurthe (149100) et la Meuse (93 866) mais devant la Moselle $(30117)^{8}$.

(6) $\mathrm{AD}$ Vosges, $6 \mathrm{~T} 1 \mathrm{~A}$ et $\mathrm{B}$, inventaires et correspondances; $\mathrm{AD}$ Vosges, $9 \mathrm{Q} 1$ à 5 bis, inventaires, états et procès-verbaux de vente du mobilier des établissements religieux, 1790-1791; BMI Épinal-Golbey, AR2-226, carton des inventaires; BM Mirecourt et Remiremont, inventaires et correspondance.

(7) Pierre Riberette, « De la Commission des monuments au Conseil de conservation », dans Dominique VARRY, Histoire des bibliothèques françaises... op. cit., p. 43.

(8) Dominique VARrY, «Les confiscations révolutionnaires », Histoire des bibliothèques françaises... op. cit., p. 20. 


\section{Les premiers inventaires}

Les premiers inventaires sont établis au printemps 1790, en application des décrets de l'Assemblée nationale des 20 février, 19 et 21 mars 1790, ratifiés par le roi le 26 mars 1790. Dans tout le département les officiers municipaux procèdent aux inventaires des « biens mobiliers, bibliothèques et archives » des établissements ecclésiastiques supprimés : trois inventaires ont lieu fin avril, une vingtaine en mai et le reste en août et septembre (notamment des établissements féminins) ${ }^{9}$. Ces inventaires sont le plus souvent sommaires et les procès-verbaux de ce printemps 1790 sont inégalement utilisables. Quelques-uns indiquent uniquement qu'il existe une bibliothèque contenant des livres, notamment dans les couvents spinaliens ${ }^{10}$. À Dommartin-lès-Ville, les officiers municipaux indiquent la répartition par format. Ceux de Mirecourt dressent deux listes, une pour les cordeliers et une pour les capucins ${ }^{11}$.

Mais l'inventaire est souvent incomplet, ainsi l'administrateur de Darney, chargé d'inventorier les livres des récollets recense correctement 29 ouvrages ( 372 volumes) et néglige 402 volumes « sous différents formats et différentes matières qu'il serait trop long d'écrire séparément vu le peu d'importance de la majeure partie de ces ouvrages $\rangle^{12}$. Pour les administrateurs, la question se pose de la qualité des livres. Sont-ils intéressants ou ne sont-ils que des "fatras de mauvais sermonaires, de vie de saints et autres puérilités $\rangle^{13}$ ? Ces remarques répétées témoignentelles, de la part des officiers chargés de ces inventaires, qui sont pourtant assez modérés en ce début de Révolution, de sentiments ou sensibilités détachés de la religion catholique, ou de la conviction que tous ces ouvrages de piété n'ont guère de valeur et sont très répétitifs? La question se pose au moment des inventaires, elle se reposera plus tard au moment du transfert au chef-lieu de district.

Ces inventaires des biens mobiliers du printemps 1790 sont vérifiés, par des administrateurs des districts, en décembre 1790 puis au cours de l'année 1791 avant la vente des biens ecclésiastiques. Le plus souvent,

(9) AD Vosges, 9 Q 1 à 5 bis, inventaires, états et procès-verbaux de vente du mobilier des établissements religieux.

(10) Ibid., 9 Q 2, inventaire des biens meubles des capucins de Mirecourt, 30 avril et des annonciades célestes d'Épinal, 3 mai 1790.

(11) Ibid., $9 \mathrm{Q} 4$, catalogue de la bibliothèque des capucins de Mirecourt $9 \mathrm{Q} 1$ et $13 \mathrm{H} 1$, catalogues de la bibliothèque de Dommartin-lès-Ville.

(12) Ibid., 6 T 1 A, inventaires des livres des récollets de Darney, 17 septembre 1791.

(13) Ibid., lettre du procureur syndic de Darney au procureur général syndic, 10 mai 1791. 
les nouveaux inventaires apportent peu de renseignements nouveaux sur les bibliothèques, les administrateurs mentionnent que les bibliothèques inventoriées en mai sont dans le même état ${ }^{14}$. Pourtant, ces inventaires et ces vérifications permettent de dresser un tableau, proche de l'exhaustivité, des bibliothèques ecclésiastiques des Vosges. Ces données ont été comparées à celles de Jean Kastener ${ }^{15}$. Pour cinquante-quatre établissements religieux ayant une bibliothèque, le total des livres recensés globalement ou en détail, s'élève à plus de 70000 volumes, résultat supérieur à celui calculé à partir des données de Kastener (61 000 volumes).

\section{Scellés et bibliothèques particulières}

Les décrets qui demandent à la fin de 1790 et au début de 1791 de vérifier les inventaires, ordonnent aussi d'apposer les scellés sur les portes et fenêtres des bibliothèques. C'est le cas dans le district de Lamarche où les scellés sont posés entre le 29 décembre 1790 et le 14 janvier $1791^{16}$. Cependant les cordeliers de Neufchâteau et les prémontrés de Parey-sousMontfort demandent à conserver l'accès à leurs livres.

La question se pose aussi des bibliothèques qui se trouvent dans les cellules ou appartements des religieux. Dans le couvent des cordeliers de Mirecourt, trois bibliothèques particulières sont mentionnées; elles contiennent 488 volumes dont $30 \%$ de volumes profanes, bien davantage que dans celle du couvent (voir tableau ci-après).

\section{Des inventaires plus précis établis par des ecclésiastiques}

Des inventaires plus précis sont demandés pour réaliser l'inventaire général de France. L'administration départementale, relayant les circulaires des comités, presse les administrations de faire les inventaires selon des instructions imprimées. Celle du 15 mai 1791, signée des comités réunis d'administration ecclésiastique et d'aliénation des biens nationaux, demande d'établir des fiches ${ }^{17}$.

(14) Ibid.,9 Q 4, recollement de l'inventaire de la bibliothèque des cordeliers de Neufchâteau, 30 décembre 1790 .

(15) Ibid., BR 4752; Jean KASTENER, Chapitres, abbayes, prieurés et couvents du département des Vosges au moment de leur suppression en 1790.

(16) Ibid., $6 \mathrm{~T} 1 \mathrm{~A}$, lettre du procureur syndic de Lamarche au procureur général syndic, 12 août 1791 .

(17) Ibid., $6 \mathrm{~T} 1 \mathrm{~B}$, Instruction pour procéder à la confection du catalogue de chacune des bibliothèques... 15 mai 1791 . 
Ces instructions sont répercutées très tôt par le procureur général syndic du département, qui, par une circulaire datée du 6 juin 1791 rappelle les textes qui « imposent aux directoires des districts de faire des catalogues des livres manuscrits, tableaux, gravures et autres objets de ce genre qui se trouveront dans les bibliothèques et cabinets des maisons des communautés supprimées [...] ou de faire un recollement sur les catalogues ou inventaires qui auraient été faits » et il demande aux districts de s'occuper sans relâche à la formation des catalogues de livres. Mais le procureur général syndic envisage le cas où la tâche s'avérerait trop complexe :

« Si des bibliothèques qui existent dans l'arrondissement de votre district sont assez considérables pour demander de ceux qui seront chargés des opérations prescrites des connaissances plus étendues [...] que n'en ont les sujets sur lesquels votre district serait porté de fixer son choix, je vous prie de m'en prévenir afin que je puisse en instruire le directoire du département et qu'il puisse vous indiquer quelqu'un en état de se charger de cette besogne importante $»^{18}$.

Le département des Vosges met donc en application la méthode des fiches cartonnée et il est de ceux qui ont accompli le travail de façon très consciencieuse. Un tableau dressé par Dominique Varry récapitule le nombre de cartes envoyées à Paris et conservées aux Archives nationales et montre que les administrateurs vosgiens se sont fait obéir ${ }^{19}$ :

\begin{tabular}{|l|c|c|}
\hline \multicolumn{1}{|c|}{ Département } & $\begin{array}{c}\text { Nombre de bibliothèques } \\
\text { inventoriées }\end{array}$ & Nombre de fiches \\
\hline Vosges & 53 & 42529 \\
\hline Meurthe & 65 & 34470 \\
\hline Meuse & 26 & 6812 \\
\hline Moselle & 15 & 1924 \\
\hline Total France & 2348 & 884127 \\
\hline
\end{tabular}

Ils se sont adressé aux personnes qui apparaissaient les plus compétentes, des anciens ecclésiastiques: à Étival, «Nicolas Briot ancien curé de Saint-Michel », dresse le catalogue et dépose le 13 février 1792

(18) Ibid., $6 \mathrm{~T} 1 \mathrm{~A}$, lettre du procureur général syndic aux procureurs syndics des Vosges, 6 juin 1791.

(19) Dominique VARrY, « Les confiscations révolutionnaires », op. cit., p. 23. 
« une boite contenant 3658 cartes sur lesquelles sont marquées les titres des livres de la bibliothèque de la ci-devant abbaye d'Étival, un volume relié contenant le catalogue de tous les livres de la dite bibliothèque au nombre de $7600 »$. Il demande pour ses six mois et douze jours de travail, et deux mois de son aide, 857 livres 14 sols, qui lui sont payées ${ }^{20}$. À Moyenmoutier, le catalogue est dressé par «Joseph Benoît Didelot, prêtre, ci-devant bénédictin en vertu d'une commission à lui donnée par le département en $1791 »$.

Les livres des bibliothèques du district de Remiremont (SaintMont, Hérival, capucins) ont été classés par ordre alphabétique des noms d'auteurs, ou « du titre signifiant », le catalogue a été envoyé à l'Assemblée nationale le 15 mai 1792 et les cartes, au département, le $1^{\text {er }}$ juin 1792. Sur les cartes la mention du lieu d'origine a été indiquée ${ }^{21}$.

Mais ce travail d'inventaire, s'il est commode à réaliser et demande peu de connaissances bibliographiques, subit des critiques comme cellesci, formulées à Senones, en l'an IX :

"Ces livres sont classés sans aucun ordre des matières, ni de distinction d'auteurs, le catalogue au contraire est fait sans égard au chiffre numérique et les ouvrages portés sur différents cahiers, cela présente souvent beaucoup de difficultés pour les retrouver, en faire la vérification [...] On veut bien croire que les commissaires chargés de la rédaction de ces catalogues avaient leur marche tracée et qu'ils l'ont suivie jusqu'à un certain point, mais pour rendre leur travail parfait, faciliter la vérification et l'exempter de toutes méprises, il fallait de leur part reformer un catalogue général en y rapportant les ouvrages par ordre de numéro d'après les fiches, et jusque-là la besogne qu'ils ont faite sera toujours incomplète $»^{22}$.

Ailleurs, cette instruction arrive trop tard pour toute une série de bibliothèques qui ont déjà été inventoriées. Ainsi, le procureur syndic du district de Saint-Dié accuse réception le 8 juin 1791 des instructions mais fait remarquer "qu'on s'occupe depuis longtemps de la formation des catalogues, l'ouvrage avance et nous espérons qu'il

(20) Georges Baumont, " Notice historique de la bibliothèque municipale de Saint-Dié », op. cit., p. 247; le catalogue se trouve à la BMI Épinal-Golbey, MS 84.

(21) Ibid., $6 \mathrm{~T} 1 \mathrm{~B}$, catalogue des livres des bibliothèques du district de Remiremont, $1^{\mathrm{er}}$ juin 1792. an IX.

(22) Ibid., compte rendu de Gœury sur l'inventaire de la bibliothèque de Senones, 9 floréal 
sera bientôt fini $»^{23}$. Un mois plus tard, son suppléant envoie l'état des livres imprimés et des manuscrits des établissements séculiers et réguliers supprimés, mais fait remarquer que celui de Moyenmoutier est inachevé car il est en contradiction avec les instructions du 15 mai. Il demande s'il faut «tout recommencer selon les instructions », ce qui ne pourra se faire car Richard est trop âgé et il sera difficile de le remplacer, « sauf à employer Briot, ex-curé de Saint-Michel qui réunit aux connaissances locales, les autres qualités requises par l'instruction même $»^{24}$.

La mise à disposition de la nation des biens ecclésiastiques s'est donc accompagnée dans les Vosges d'une saisie des bibliothèques de ces ordres, d'établissements d'un ou plusieurs inventaires réalisés plus ou moins scrupuleusement par les administrateurs ou par des ecclésiastiques acceptant les transformations révolutionnaires. Ces inventaires s'inscrivent aussi dans le cadre de l'inventaire général voulu par la Constituante. Cependant ils ne sont pas tous conservés et certains se trouvent encore dans les bibliothèques municipales, ce qui est le cas à Épinal, Neufchâteau et Mirecourt. Il faudrait aller les rechercher dans ces bibliothèques et les dépouiller systématiquement, comme je l'ai fait à Mirecourt, pour connaître la nature des ouvrages. Dans un premier temps, en 1790 et au début de 1791, les livres restent dans des monastères, mais la vente de ceux-ci amène la nécessité de regrouper les bibliothèques au chef-lieu de district.

\section{Le regroupement des bibliothèques}

Les bibliothèques se trouvant dans des établissements destinés à être vendus, ne peuvent demeurer sur place et leur transfert vers des «dépôts littéraires » est ordonné dès le printemps 1791. Mais cette décision pose plusieurs problèmes : quand opérer le transfert? Que transférer? Où mettre les livres? Qui paiera? Faut-il transférer les très grandes bibliothèques situées dans la montagne? Faut-il transférer les livres qui sont considérés comme sans intérêt? De plus, les municipalités et administrations de district n'acceptent pas volontiers le départ

(23) Ibid., lettre du procureur syndic du district de Saint-Dié au procureur général syndic des Vosges, 8 juin 1791.

(24) Ibid., lettre du procureur syndic suppléant du district de Saint-Dié au procureur général syndic des Vosges, 4 juillet 1791. 
de ce qu'ils considèrent comme des richesses littéraires vers d'autres villes.

Fig. 2 : Carte des bibliothèques des Vosges avant leur regroupement.

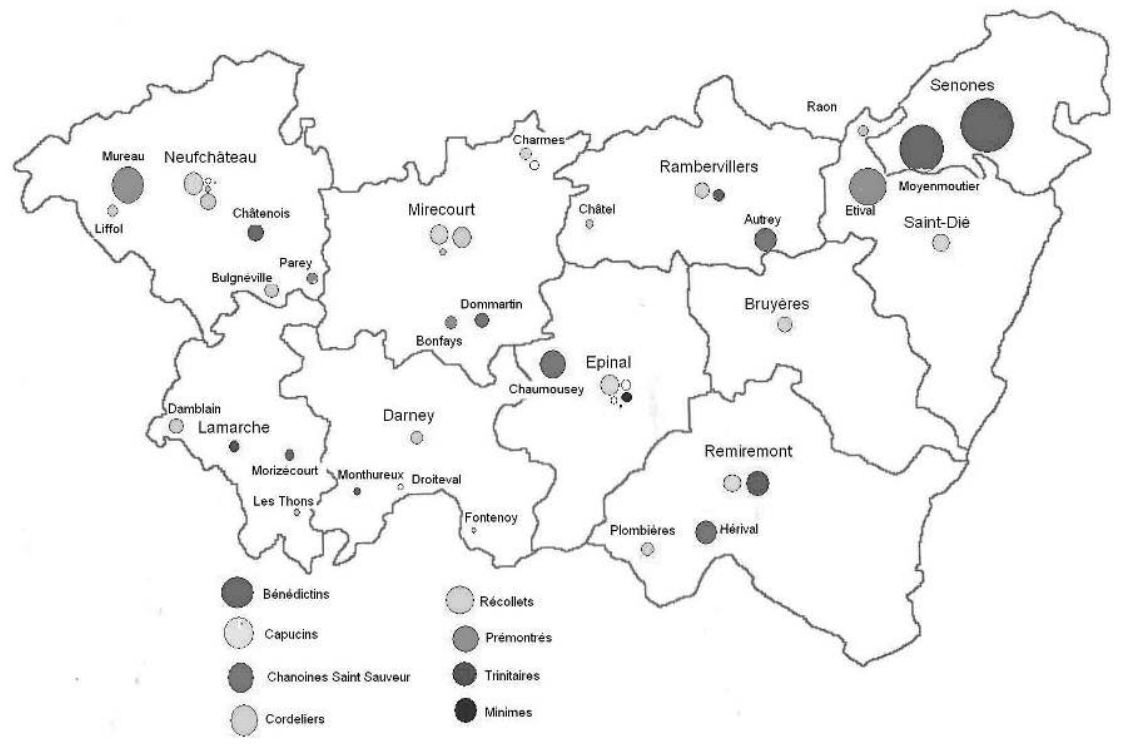

\section{Les transferts vers les chefs lieux de district}

Bien que la loi qui organise les bibliothèques de district date du 8 pluviôse de l'an II, des « dépôts littéraires » sont réalisés dès 1791 et ils portent très tôt le nom de «bibliothèques nationales ». Les administrateurs reprennent les arguments nationaux pour justifier la conservation des livres et la création de ces dépôts dans les districts :

« Les administrateurs du district [de Saint-Dié], considérant combien le souvenir des efforts de l'esprit humain est capable d'entretenir les goûts des arts et de la culture des belles lettres, et combien il serait avantageux d'en faciliter les moyens en multipliant les connaissances par la correspondance des pensées de ceux qui nous ont précédé, ne croit pas qu'il y ait un moyen plus propre de seconder le goût général du public que d'établir une bibliothèque dans chaque district.

Ces dépouilles monastiques sont précieuses [...] par leur richesse littéraire accumulée à grands frais [...] Les belles lettres, les sciences et les arts, l'instruction et même les mœurs feraient des progrès plus rapides, 
aussi serait-ce un mouvement de sagesse et de prévoyance, car jamais le goût des arts n'a été plus répandu, jamais la raison et l'esprit n'ont été si recherchés, et jamais les lettres n'ont été plus cultivées, il faut donc fournir $[\ldots]$ à l'intention de si louables dispositions $»^{25}$.

L'administration du district de Saint-Dié demande, dès septembre 1790, qu'on réunisse dans chaque chef-lieu de district un « fond de bibliothèque qui serait formé d'une partie des livres des monastères supprimés, afin de conserver dans un dépôt public ces débris précieux dont la dispersion serait peu utile aux particuliers et d'un profit à peu près nul pour la nation $\gg^{26}$. En 1791, ce district met en réserve le couvent de capucins pour établir cette bibliothèque et une école secondaire, et il y regroupe les trois bibliothèques ecclésiastiques de Saint-Dié (capucins, séminaire et chapitre) et celle des cordeliers de Raon ${ }^{27}$. Il demande en octobre que les bibliothèques de Moyenmoutier et d'Étival soient « translatées » au cheflieu de district, mais le projet n'aboutit pas $^{28}$.

Par contre, le transfert vers le chef-lieu de district de Neufchâteau des livres de tous les établissements du district s'opère en avril 1791 : les livres des prémontrés de Mureau, du prieuré bénédictin de Châtenois, du couvent des cordeliers de Liffol, des récollets de Bulgnéville et des prémontrés de Parey-sous-Montfort, sont chargés sur des « voitures agricoles », conduites au chef-lieu de district et placés dans une maison appartenant à la municipalité, l'ancien presbytère ${ }^{29}$. Dans le district de Remiremont, le transfert de la bibliothèque d'Hérival a lieu le 29 décembre 1790, alors que les bénédictins du Saint-Mont transfèrent seuls leurs livres vers leur maison de Remiremont. Dans le district de Mirecourt, les bibliothèques des prémontrés de Bonfaÿs, des chanoines réguliers de Dommartin-lèsVille, des capucins et des clarisses de Mirecourt sont transférées dans le couvent des ci-devant cordeliers, probablement en avril-mai 1791. Dans le district de Bruyères, un seul dépôt existe au chef-lieu ${ }^{30}$. Dans le district de Rambervillers, les livres du prieuré d'Autrey restent sur place

(25) Ibid., 6 T 1 B, état des livres des abbayes du district de Saint-Dié, $1^{\mathrm{e}}$ juillet 1791.

(26) Pétition de Dubert et Huin administrateurs du district de Saint-Dié, cité par Georges Baumont, « Notice historique de la bibliothèque municipale de Saint-Dié », Le Pays lorrain, juin 1930, p. 242.

(27) Georges Baumont, « Notice historique de la bibliothèque municipale de Saint-Dié... op. cit., p. 248.

(28) Ibid., p. 242-243.

(29) AD Vosges, 6 T 1 B, procès-verbal de transfert des livres, mai 1791.

(30) Ibid., 6 T 1 A, lettre de l'administration municipale à l'administration centrale, 27 nivôse an VII. 
malgré la vente des bâtiments; ce n'est qu'en avril 1792 que le nouveau propriétaire, le maître de forges Colombier, demande au district d'enlever la bibliothèque; l'administration du district la transfère alors dans le couvent des bénédictines de Rambervillers ${ }^{31}$.

Fig. 3 : Carte des bibliothèques des Vosges après leur regroupement.

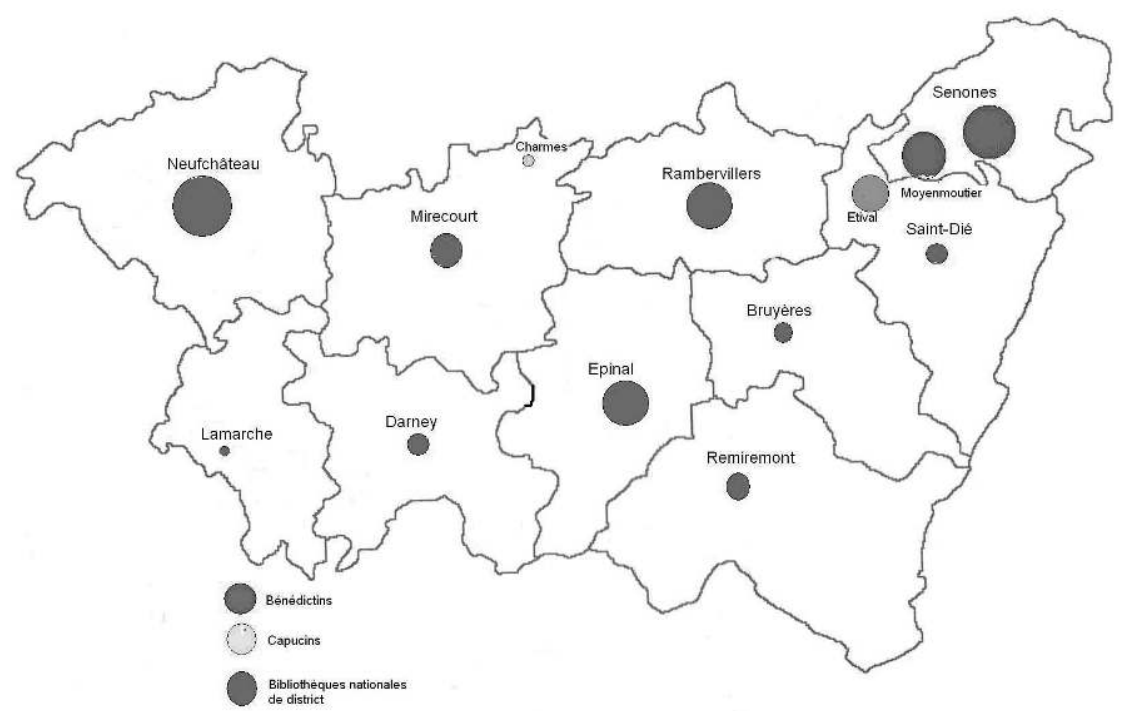

Mais très tôt se pose la question : faut-il transférer tous les livres? En valent-ils la peine? Certains administrateurs répondent par la négative et les livres de trois monastères sont vendus : à Monthureux-surSaône " à une multitude d'artisans pour apprendre à lire à la jeunesse »; à Fontenoy-le-Château et aux Thons, les livres jugés de peu d'importance sont vendus aux enchères ${ }^{32}$. Les dilapidations sont importantes dans le district de Remiremont où l'administrateur Berguam refuse de charger de nombreux livres : à Hérival, 1000 « volumes détachés, qui sont de peu de valeur, pas même pour la voiture » sont " laissés dans la maison » sous

(31) Ibid., L 729, registre des délibérations du directoire du district de Rambervillers, 26 avril 1792.

(32) Ibid., $6 \mathrm{~T} 1 \mathrm{~A}$, lettre du procureur syndic de Darney au procureur général syndic, 10 mai 1791; Ibid., 9 Q 5bis, procès-verbal de ventes des biens mobiliers du monastère des Thons, 14 février 1791 ; c'est le curé du lieu qui achète la bibliothèque pour la somme de 12 livres 10 sols. 
la garde de la municipalité33 ; à Plombières, il se « dispense de [prendre] les livres contenus dans la bibliothèque attendu qu'ils sont en très petit nombre [environ 500], de peu de valeur, qu'il y a des ouvrages détachés et enfin qu'ils ne valent pas le prix de la voiture $»^{34}$. À Neufchâteau, la bibliothèque des clarisses, «19 volumes de dévotion [...] ont été abandonnés aux dames de la maison $\gg{ }^{35}$. Cependant ces exemples de dilapidations sont assez limités et presque tous les livres sont conservés, une partie étant transférée vers les chefs-lieux de district, à des dates très différentes les unes des autres.

\section{La création et le fonctionnement des bibliothèques de district}

La loi du 8 pluviôse de l'an II qui organise les bibliothèques de district demande aux administrateurs de proposer une maison nationale, d'en prévoir l'aménagement et de fournir les inventaires dans de brefs délais... Contrairement à ce qu'affirme Hélène Richard ${ }^{36}$, des bibliothèques de district ont été installées et ont fonctionné dans la plupart des districts des Vosges.

À Mirecourt, les livres de cinq maisons religieuses sont regroupés dans la bibliothèque des cordeliers de la ville (située au premier étage du cloître, dans « deux chambres sans cheminée, prenant jour sur le cloître vers le couchant $\gg{ }^{37}$ ) et un catalogue est établi, achevé en juillet 1792, classant les livres par maison, puis par ordre alphabétique des auteurs, mais en ne prenant pas en compte « 7 à 800 volumes [...] missels, antiphonaires, pontificaux, bréviaires, une foule de prédicateurs espagnols, italiens etc. traduits en français au XVI ${ }^{\mathrm{e}}$ siècle, quelques prédicateurs du même temps, quelques livres ascétiques qui n'offrent aucun motif d'intérêt $\rangle^{38}$.

Un pointage par grands thèmes, réalisé à partir des inventaires de 1790 (cordeliers) et de 1792, montre que les livres de théologie constituent entre les trois-quarts et la quasi-totalité des ouvrages saisis dans

(33) Ibid., 9 Q 4, procès-verbal de transport des meubles d'Hérival à Remiremont, 10 février 1791.

(34) Ibid., 9 Q 5, procès-verbal de transport des meubles des capucins de Plombières, 30 mai 1791.

(35) Ibid., recollement de l'inventaire des clarisses de Neufchâteau, 20 septembre 1792

(36) Hélène Richard, "Des bibliothèques de districts aux bibliothèques municipales ", dans Dominique VARry, Histoire des bibliothèques françaises... op. cit., p. 44.

(37) AD Vosges, 4 Q Pro 26, inventaire des biens mobiliers du couvent des cordeliers de Mirecourt.

(38) BM Mirecourt, catalogue des livres de la bibliothèque des cordeliers, capucins, clarisses de Mirecourt... 25 juillet 1792. 
les bibliothèques ecclésiastiques. Rien ou presque dans les ouvrages de jurisprudence ou de sciences, bien peu d'ouvrages d'histoire, et ce sont souvent des ouvrages d'histoire ecclésiastique. Le contraste est considérable avec la bibliothèque de Charles Gabriel Canon, marquis de Ville-surIllon, saisie pour cause d'émigration et dont l'inventaire, dressé les 16 et 17 octobre 1792, révèle 1648 volumes, dont les grands livres de l'époque des Lumières : l'Histoire naturelle de Buffon en 82 volumes in- $4^{\circ}$, l'Encyclopédie de Diderot et d'Alembert, l'Histoire de Lorraine de Dom Calmet, les œuvres de Rousseau, Voltaire, Montesquieu, l'abbé Prévôt, Lesage... Un contenu tout à fait différent des bibliothèques ecclésiastiques.

Fig. 4 : Contenu des bibliothèques du district de Mirecourt, 1790-1792.

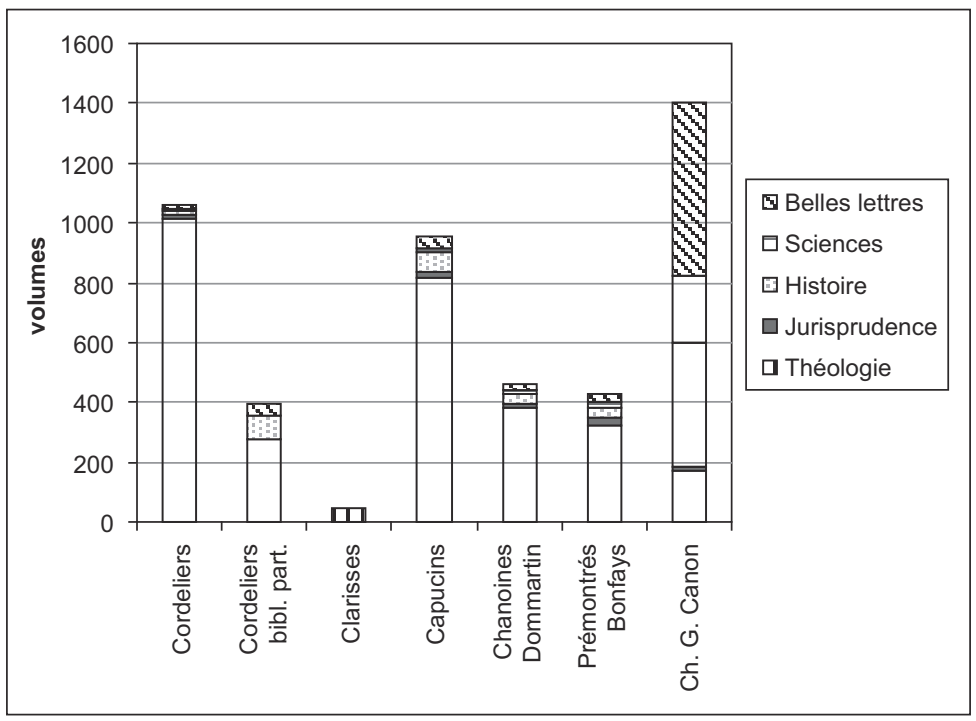

À Remiremont, la bibliothèque du district existe à la suite du regroupement des trois bibliothèques, dans deux salles de l'hospice, « un local saint, où les livres sont bien placés » ${ }^{39}$. Charles François Roussel est chargé par l'administration, à partir du 3 ventôse de l'an $\mathrm{II}^{40}$, de « veiller à la conservation et à l'arrangement de la bibliothèque

(39) AD Vosges, $6 \mathrm{~T} 1 \mathrm{~B}$, lettre du sous-préfet de Remiremont au préfet des Vosges, 11 germinal an IX.

(40) Charles François Roussel, prémontré d'Étival, vicaire constitutionnel à Girancourt en mai 1791, vicaire à Remiremont en avril 1792, sans-culotte, abdique fonction et prêtrise le 9 pluviôse an II. 
du district, de recueillir tous les livres des différentes maisons religieuses supprimées et ceux des chanoines et chanoinesses émigrés du ci-devant chapitre $»^{41}$; il forme alors une bibliothèque « considérable »; il demande, en messidor an III, une indemnité pour le temps passé et il obtient 450 livres pour les trois mois travaillés ${ }^{42}$. Il est remplacé à ce moment par Anselme Maxel qui travaille quatre-vingt dix jours depuis le 3 prairial an II et est payé 9 livres par jour ${ }^{43}$. Lui succède l'abbé Janny ${ }^{44}$, qui est chargé de former la bibliothèque de Libremont; en brumaire de l'an III, il demande à être payé 1200 livres pour cent jours de travail, mais le district ne lui accorde que 9 livres par jour, soit 900 livres $^{45}$. D'autres personnes travaillent en même temps au classement des livres, à rédiger des cartes pour la Convention et à dresser le catalogue, comme Jean Baptiste Lefebvre qui fait 1000 cartes de bibliothèque, à 2 sols la pièce, soit 100 livres $^{46}$.

La situation des autres districts est résumée dans le tableau ci-dessous :

\begin{tabular}{|c|c|c|c|}
\hline District & Bibliothécaire & Catalogue & Nombre de volumes \\
\hline Neufchâteau ${ }^{47}$ & $\begin{array}{l}\text { Herbinot, ancien } \\
\text { curé de Rouceux }\end{array}$ & 770 pages & 15906 \\
\hline Darney $^{48}$ & $\begin{array}{l}\text { Michel Maugeure, } \\
\text { ancien bénédictin } \\
\text { de Senones }{ }^{49}\end{array}$ & $\begin{array}{l}969 \text { cartes et } \\
\text { un catalogue }\end{array}$ & 2078 \\
\hline Rambervillers $^{50}$ & François Drouel & & \\
\hline Épinal ${ }^{51}$ & $\begin{array}{l}\text { Jean Baptiste } \\
\text { Ganier et Léonors } \\
\text { Piers }\end{array}$ & $\begin{array}{l}\text { catalogue de } \\
526 \text { pages }\end{array}$ & 9087 \\
\hline
\end{tabular}

(41) AD Vosges, 6 T 1 B, pétition de Roussel, 8 messidor an III.

(42) Ibid., lettre du procureur général syndic au procureur syndic de Remiremont, 27 messidor an III ; Ibid., réponse du district à la pétition de Roussel, 8 messidor an III.

(43) Anselme Maxel, bénédictin du Saint-Mont, vicaire de Vecoux en 1780, vicaire constitutionnel à Saulxures-sur-Moselotte, prête tous les serments, nommé curé du Valtin en 1803.

(44) Nicolas Janny, chanoine chapelain du chapitre de Remiremont en 1788, aumônier de la garde nationale en 1789 , prête le serment constitutionnel, abdique et dépose ses lettres de prêtrise en messidor an II, professeur à l'école centrale d'Épinal en 1796, directeur de l'école secondaire de Remiremont en 1802.

(45) AD Vosges, 6 T 1 B, pétition de Janny, 8 brumaire an III.

(46) Ibid., pétitions de Maxel et de Lefebvre, 20 nivôse et 3 ventôse an III.

(47) Ibid., liasse Neufchâteau. 
Il est clair que les bibliothèques nationales ont été créées grâce au regroupement des livres des différentes communautés religieuses, sauf pour le district de Saint-Dié, auxquels se sont ajoutés les livres des émigrés, nobles ou ecclésiastiques. Un gros travail de tri et de confection de catalogues a été fait par des ecclésiastiques acceptant la Révolution, ayant abdiqué au moment de la déchristianisation et s'étant mis au service des districts, qui ont accepté avec empressement ces personnes qualifiées et les ont rémunérés correctement. Cependant il s'agit fondamentalement d'un travail de réorganisation, de classement et de sauvegarde et il ne semble pas que ces bibliothèques aient été ouvertes au public, avec des activités de prêt. Nous n'avons pas trouvé de traces de telles activités.

\section{La réorganisation sous le Directoire, bibliothèques centrale et municipales}

Après la suppression des districts par la constitution de l'an III, la question du maintien de ces bibliothèques de district se pose et le ministre de l'Intérieur donne une instruction, le 7 floréal an IV, pour créer des bibliothèques à côté des écoles centrales et d'autres établissements scolaires ${ }^{52}$.

Face à ces instructions, quelle attitude eut l'administration du département des Vosges? A-t-elle pris une partie des livres des dépôts ou a-t-elle tout regroupé à Épinal pour le service de l'école centrale du département? La tentation était forte pour l'administration départementale, mais les municipalités des principales villes vosgiennes s'appuient sur l'instruction qui prévoit aussi la possibilité de création ou de maintien de bibliothèque dans les petites villes sans école, et profitent de cette brèche dans le processus de regroupement.

\section{La bibliothèque établie près de l'école centrale à Épinal}

À Épinal, la bibliothèque de l'ancien district, reste «sans surveillance », ses livres pouvaient «s'avarier faute de soin ». L'adminis-

(48) Ibid., 6 T 1 A.

(49) Michel Maugeure, bénédictin de Senones, vicaire épiscopal constitutionnel de juin 1791 à septembre 1792, puis curé de Darney jusqu'à son abdication en juillet 1794 .

(50) AD Vosges, L 729.

(51) BMI Épinal Golbey, Ms 21.

(52) Voir Hélène RichARD, « Des bibliothèques de districts aux bibliothèques municipales », dans Dominique VARRY, Histoire des bibliothèques françaises... op. cit., p. 50. 
tration départementale nomme alors, le 3 frimaire an IV, Dominique François Louis Chenin ${ }^{53}$, pour reclasser, ranger les livres et faire un nouveau catalogue qui sera celui de la bibliothèque de l'école centrale ${ }^{54}$. Il touche le même salaire que les professeurs, 2000 francs par an, fait partie de l'assemblée générale de l'école centrale et participe aux prises de décisions au même titre que les professeurs ${ }^{55}$.

L'école centrale ouvre le $1^{\mathrm{er}}$ messidor an IV et la bibliothèque est mise en place ${ }^{56}$. Elle est partie intégrante de la pédagogie nouvelle, devant fournir des instruments de travail aux élèves de l'école ${ }^{57}$. Mais quelle est la réalité de cette bibliothèque? Les données sont contradictoires, Chenin, dans une lettre du 14 frimaire an VII au ministre de l'Intérieur, déclare qu'il a fait, à ses frais, l'acquisition des premiers livres achetés par la bibliothèque ${ }^{58}$. Se met-elle en place si lentement que les professeurs doivent acheter eux-mêmes les livres dont ils ont besoin ${ }^{59}$ ? Pourtant les professeurs de l'école centrale, Sébastien Gérardin, professeur d'histoire naturelle, Jean Bédel, professeur de mathématiques, nommés par commission, ont fait le tour des bibliothèques de la montagne, en prairial an IV, pour choisir les « tableaux, cartes, livres, machines et autres objets de nature à entrer dans une collection d'histoire naturelle $\rangle^{60}$, et l'inventaire des livres de l'école centrale d'Épinal, en l'an IV, montre des fonds très importants :

\begin{tabular}{|c|c|c|c|c|c|c|}
\hline \multicolumn{6}{|c|}{ Nombre et formats des livres de en l'an IV ${ }^{61}$} \\
\hline & In folio & In quarto & In octavo & In 12 & $\begin{array}{l}\text { Total } \\
\text { ouvrages }\end{array}$ & $\begin{array}{l}\text { Total } \\
\text { volumes }\end{array}$ \\
\hline École centrale & 1282 & 1380 & 6408 & 5738 & 9070 \\
\hline
\end{tabular}

Les livres, provenant des maisons ecclésiastiques et d'émigrés du district d'Épinal, sont classés, conformément aux instructions ministé-

(53) Dominique François Louis Chenin, prieur de Chaumousey, principal du collège d'Épinal, abdicataire de ses fonctions et de son état ecclésiastique en 1794. Il ne reprend pas de fonctions ecclésiastiques en 1795 .

(54) Boniface Georgin, L'enseignement secondaire à Épinal... op. cit., t. XVIII, 1930, p. 133.

(55) Martine Claude, Trois écoles centrales lorraines..., op. cit., p. 60.

(56) Ibid., p. 20.

(57) Ibid., p. 93.

(58) Boniface Georgin, Ibid., t. XX, 1931, p. 65; AN F 17 A 1344-31.

(59) Martine Claude, Trois écoles centrales lorraines..., op. cit., p. 30 et 54.

(60) Georges BAumont, « Notice historique de la bibliothèque municipale de Saint-Dié », op. cit., p. 247.

(61) AD Vosges, 6 T 1 A, inventaire des livres du dépôt de l'école centrale, an IV. 
rielles, en fonction des sections de l'école centrale. On peut noter l'intérêt porté par François de Neufchâteau, qu'il soit ministre de l'Intérieur ou président de l'administration départementale, au fonctionnement de l'école centrale et de sa bibliothèque.

\section{Conserver les bibliothèques des ci-devant districts?}

En fait, le sort des bibliothèques des ci-devant districts varie d'un lieu à l'autre et les sources relatives à ces transferts éventuels, sont ici encore très incomplètes.

À Mirecourt, un catalogue est établi, probablement au début de l'an IV, il regroupe par thème les livres des couvents et des émigrés rassemblés en 1792. La bibliothèque est passée de 4000 volumes à plus de 6000, par l'adjonction des livres des émigrés. Le catalogue ne fait plus mention des origines des livres, mais il les classe par grands ensembles thématiques, où l'on note le recul, relatif, des livres de théologie :

\begin{tabular}{|l|c|c|c|c|c|c|c|}
\hline Bibliothèque de Mirecourt sous le Directoire \\
\hline & in-folio & in-4 & in- $8^{\circ}$ & in-12 & in-18 & total & \% \\
\hline Théologie (lettres A à F) & 310 & 234 & 954 & 1142 & 104 & 2744 & $\mathbf{4 5 , 4}$ \\
\hline Jurisprudence & 31 & 38 & 45 & 23 & 24 & 161 & $\mathbf{2 , 7}$ \\
\hline Histoire & 62 & 118 & 281 & 750 & 45 & 1256 & $\mathbf{2 0 , 8}$ \\
\hline Sciences et arts & 106 & 237 & 235 & 345 & 24 & 947 & $\mathbf{1 5 , 7}$ \\
\hline Belles lettres & 10 & 38 & 204 & 571 & 114 & 937 & $\mathbf{1 5 , 5}$ \\
\hline Total & 519 & 665 & 1719 & 2831 & 311 & 6045 & 100 \\
\hline
\end{tabular}

Dans le ci-devant district de Neufchâteau, l'administration du département demande, en l'an $\mathrm{V}$, outre l'envoi à Épinal du catalogue de la bibliothèque du district, le transfert de celle-ci au chef-lieu de département. L'administration municipale de canton montre l'importance et le coût de ce transfert, souligne que de nombreux livres sont en double, qu'ils ne sont pas tous intéressants et qu'il faudrait faire un tri avant un transfert ${ }^{62}$. Elle argumente aussi sur le fonds : il faut laisser « une certaine quantité de livres, des ouvrages doubles ou triples pour servir à l'instruction publique ». La bibliothèque de Neufchâteau

(62) AD Vosges, $6 \mathrm{~T} 1 \mathrm{~B}$, lettre de l'administration de canton à l'administration centrale des Vosges, 2 prairial an $\mathrm{V}$. 
ne quitte pas la ville, elle y est encore au début du Consulat mais plusieurs plaintes dénoncent les «abus» et les «vols» de livres ${ }^{63}$, alors qu'un certain nombre de livres ont été « rendus à des prêtres déportés ou à leurs héritiers, et d'autres ont été envoyés au Comité de l'Instruction publique $»^{64}$.

En l'an VII, l'administration de la municipalité de canton de Saint-Dié propose de nouveau à l'administration départementale, de créer à Saint-Dié une bibliothèque regroupant les livres des ci-devant maisons religieuses. Elle argumente sur «le mauvais état où elles se trouvent et le désordre qui y règne », et la nécessité de sauver "ces dépôts précieux $»^{65}$. Mais les transferts ne se font pas et les grandes bibliothèques ecclésiastiques restent à Moyenmoutier, Senones et Étival.

Finalement, le projet directorial de rassembler au chef-lieu du département, près de l'école centrale, les livres les plus intéressants des districts a échoué dans les Vosges. S'appuyant sur les incertitudes des réglementations, les autorités des municipalités de canton ont su conserver l'héritage des districts et peu de livres ont gagné Épinal et son école centrale. Pendant cette période, les fonds se sont un peu ouverts à la lecture, des livres ont été sortis et certains n'ont pas regagné les bibliothèques. Cela a permis au nouveau régime consulaire de faire porter la responsabilité des faiblesses d'organisation aux bibliothécaires précédents et surtout au régime directorial qu'il est de bon ton de critiquer ${ }^{66}$.

\section{Sous le Consulat : vérifications, restitutions et transferts}

Le changement de régime, la suppression des écoles centrales par la loi du 11 floréal an XI ( $1^{\text {er }}$ mai 1802), et leur remplacement par des lycées, entraînent la suppression des bibliothèques liées aux écoles centrales. Le décret du 8 pluviôse an XI (28 janvier 1803) prévoit que l'on puisera dans les bibliothèques des anciennes écoles centrales pour consti-

(63) Ibid., lettre du sous-préfet de Neufchâteau au préfet des Vosges du 3 germinal an IX.

(64) AD Vosges, $6 \mathrm{~T} 1 \mathrm{~B}$, lettre de l'administration de canton à l'administration centrale des Vosges, 2 prairial an V. an VII.

(65) Ibid., lettre de l'administration de la municipalité de canton de Saint-Dié, 17 ventôse tôse an IX.

(66) Ibid., 6 T 1 A, lettre du maire de Bruyères, Georgel, au préfet des Vosges, 29 ven- 
tuer celles des lycées. Les volumes qui resteront devront être conservés avec soin, précisait le rapport préliminaire à ce décret, afin de constituer des bibliothèques mises sous la responsabilité des municipalités. Celles-ci nommeront un conservateur, dont le traitement sera payé par la commune, et qui devra faire un inventaire des livres. Le gouvernement se réserve la possibilité de puiser dans ces bibliothèques pour d'autres « établissements d'instruction publique $»^{67}$. Dans les Vosges, ces instructions sont mises en application et le préfet pose de nouveau la question du transfert total ou partiel vers Épinal des livres des bibliothèques des ci-devant districts. Il demande aussi que l'on rende leurs livres aux émigrés amnistiés et aux prêtres déportés de retour. Ces restitutions et transferts nécessitent de nouveaux inventaires systématiques par thèmes qui seront confrontés à ceux réalisés en 1790-1791. Cette période est aussi celle d'un transfert de responsabilité, des municipalités de cantons aux municipalités communales.

\section{Les vérifications et l'achèvement des inventaires}

Menées dès l'an IX, sur ordre du préfet, les vérifications font apparaitre de nombreuses disparitions de livres après que l'on ait soulevé la question de l'absence des catalogues qui ont été envoyés à Épinal, situation qui retarde les vérifications.

À Mirecourt, le dépôt « avait peu d'ordre, personne n'était chargé de le garder et tous les livres étaient entassés pêle-mêle », déclare en l'an IX le sous-préfet Cera-Lebrun lorsqu'il arrive dans la ville (pourtant les catalogues établis sous le Directoire ne laissent pas cette impression); il a le projet de "rendre ce dépôt utile aux citoyens de l'arrondissement en ouvrant la bibliothèque au public », après avoir établi un catalogue ${ }^{68}$. Il nomme François Clément comme bibliothécaire en messidor an IX; celui-ci travaille un an et demi pour établir un quatrième catalogue remarquablement précis et bien présenté par catégorie de livres ${ }^{69}$ :

(67) Hélène RichARD, « Des bibliothèques de districts aux bibliothèques municipales », op. cit., p. 56.

(69) Ibid., 6 T 1 A, lettre de Clément, bibliothécaire, au préfet des Vosges, 25 frimaire an XI. 


\begin{tabular}{|l|c|c|c|c|c|c|c|}
\hline \multicolumn{1}{|c|}{ Les livres de la bibliothèque de Mirecourt en nivôse an XI, ADV 6 T 1 A } \\
\hline & in-folio & in-4 & in- $8^{\circ}$ & in-12 & in- $18^{\circ}$ & total & \% \\
\hline Théologie & 271 & 217 & 1053 & 1357 & 84 & 2982 & $\mathbf{5 0 , 7}$ \\
\hline Jurisprudence & 38 & 23 & 48 & 32 & 11 & 152 & $\mathbf{2 , 6}$ \\
\hline Histoire & 131 & 143 & 176 & 714 & 11 & 1177 & $\mathbf{2 0}$ \\
\hline Sciences et arts & 27 & 95 & 248 & 307 & 5 & 682 & $\mathbf{1 1 , 6}$ \\
\hline Belles lettres & 26 & 35 & 228 & 574 & 26 & 889 & $\mathbf{1 5 , 1}$ \\
\hline Total Mirecourt & 493 & 513 & 1755 & 2784 & 137 & 5682 & $\mathbf{1 0 0}$ \\
\hline
\end{tabular}

Cet inventaire est dans la continuité de celui du Directoire, avec des résultats assez semblables : la prépondérance des livres de théologie et de religion est encore évidente, mais ils ne représentent que 50,7\% de l'ensemble, les livres d'histoire forment 20\% (seulement $4 \%$ de livres d'histoire religieuse), les sciences sont bien représentées avec notamment les 36 volumes de l'Encyclopédie, le droit est très peu présent, ce qui est étonnant dans un ci-devant chef-lieu de bailliage. Deux autres dépôts existent encore dans l'arrondissement de Mirecourt, celui de Charmes qui contient le « peu de livres » des capucins de la ville ${ }^{70}$ et celui de Darney qui a une « collection peu intéressante » et qui « a beaucoup souffert $»^{71}$. Face à cette situation, le sous-préfet suggère de regrouper tous les livres à Mirecourt, mais ce transfert ne semble pas avoir été réalisé.

Dans l'arrondissement de Saint-Dié, les bibliothèques ecclésiastiques sont toujours réparties dans quatre dépôts, malgré les nouvelles demandes de la municipalité de Saint-Dié et les propositions du ministre de l'Instruction publique $^{72}$. Mais un incendie ravage en vendémiaire an XI le monastère d'Étival, les livres de la bibliothèque sont jetés dans le jardin et le sous-préfet, arrivé sur place, sauve les livres et obtient leur transport et leur classement à Saint-Dié ${ }^{73}$. La bibliothèque de Saint-Dié est alors aménagée au second étage de l'hôtel de ville et les boiseries d'Étival y sont installées. Ces travaux sont achevés le 11 vendémiaire

(70) Ibid., lettres du sous-préfet de Mirecourt au préfet des Vosges, 18 pluviôse an X.

(71) Ibid.

(72) Georges Baumont, « Notice historique de la bibliothèque municipale de Saint-Dié », op. cit., p. 244.

(73) AD Vosges, 6 T 1 B, lettres du sous-préfet de Saint-Dié au préfet des Vosges, 4 vendémiaire an XI. 
an $\mathrm{XII}^{74}$. À Neufchâteau et à Bruyères, les bibliothèques sont réorganisées $^{75}$.

\section{Les restitutions aux émigrés amnistiés}

Les livres des émigrés, ecclésiastiques ou laïcs, ont été confisqués comme leurs autres biens, mais ils n'ont pas été mis en vente. Ils ont rejoint les bibliothèques de district. Cependant, lorsque les émigrés rentrent après avoir été amnistiés, ils réclament la restitution de leurs livres. C'est le cas à Bruyères où les curés déportés et rentrés, Thomas, Durand et Febvrel, récupèrent près de 400 livres le 22 germinal an III $^{76}$. À Mirecourt, le catalogue dressé pendant le Directoire contient la « liste des livres [...] rendus aux citoyens Vernet, Salle, Tassard et Rellot » prêtres qui avaient été déportés en septembre 1792. Cette liste de livres s'élève à 1038 volumes dont 23 in-folio, 128 in-quarto, 221 in-octavo, 575 in- $12^{\circ}$ et 108 in $-16^{\circ}$ ou in $-18^{\circ}$.

À Neufchâteau, à Rambervillers et à Épinal des prêtres réfractaires demandent la mainlevée de leurs livres et leur restitution. Les réponses du préfet sont presque toujours dilatoires ou négatives ${ }^{78}$.

\section{Triage, transferts et ventes}

Les regroupements opérés en faveur des chefs-lieux de districts, puis d'arrondissements, entraînaient la multiplication des livres en double ou triple exemplaire. Très tôt est venue l'idée d'échanger entre les bibliothèques ces livres ou de les vendre. Cette opération systématique se développe dans toutes les bibliothèques sous le Consulat et l'Empire. Le préfet des Vosges nomme Chenin, bibliothécaire en second d'Épinal, pour faire le tri dans les bibliothèques de Moyenmoutier et de Senones afin d'envoyer à Épinal les ouvrages les plus dignes d'intérêt ${ }^{79}$.

(74) Ibid., p. 245.

(75) Ibid., lettre du maire de Neufchâteau au sous-préfet, 6 floréal an XI; 6 T 1 A, catalogue des livres de la bibliothèque de Bruyères, 19 messidor an VIII

(76) Ibid., $6 \mathrm{~T} 1 \mathrm{~A}$, catalogue des livres des émigrés, germinal an III.

(77) BM Mirecourt, catalogue des livres de la bibliothèque [sans date].

(78) AD Vosges, $6 \mathrm{~T} 1 \mathrm{~B}$, pétition de Marteau, 23 frimaire an XI; pétition de Bocquel et réponses du préfet, 7 vendémiaire et 11 brumaire an XI; pétition de Tarillon et réponses du préfet, 21 fructidor an $\mathrm{X}$ et 11 brumaire an XI; pétition de Gérard et réponse du juge de paix, vendémiaire an XI.

(79) BMI, AR2-226, liasse de Moyenmoutier. 


\begin{tabular}{|l|l|c|c|c|c|c|c|c|}
\hline \multicolumn{10}{|c|}{ Livres à transférer à Épinal } \\
\hline \multirow{2}{*}{ Bibliothèques } & \multicolumn{2}{|c|}{ in-folio } & \multicolumn{2}{|c|}{ in-4 } & \multicolumn{2}{|c|}{ in- $8^{\circ}$ et in-12 } & \multicolumn{2}{|c|}{ Total } \\
\cline { 2 - 10 } & Ouvr. & Vol. & Ouvr. & Vol. & Ouvr. & Vol. & Ouvr. & Vol. \\
\hline Moyenmoutier & 383 & 721 & 351 & 613 & 677 & 994 & 1411 & 2328 \\
\hline $\begin{array}{l}\text { Total Senones et } \\
\text { Moyenmoutier }\end{array}$ & 639 & 1375 & 595 & 1243 & 991 & 1849 & 2325 & 4468 \\
\hline
\end{tabular}

De même la ville de Saint-Dié vend, en septembre 1806, pour 1100 francs de livres à un libraire de Lunéville et acquiert 130 ouvrages formant 502 volumes par échanges ou achats. En juin 1807, la bibliothèque de Saint-Dié dresse une liste de 68 ouvrages (193 volumes) à prendre à Moyenmoutier et de 52 ouvrages ( 269 volumes) à prendre à Senones. Ces livres, de grande valeur, arrivent à Saint-Dié en décembre ${ }^{80}$.

L'Église reconstituée après le Concordat demande la restitution de livres, ou du moins la mise à disposition de livres nécessaires au culte pour constituer des bibliothèques. L'évêque de Nancy s'appuie, pour les obtenir, sur une lettre de Chaptal, ministre de l'Intérieur, qui autorise les ecclésiastiques désignés par l'évêque à chercher «les ouvrages nécessaires à l'exercice de [ses] fonctions; quand le catalogue en sera dressé, veuillez me l'adresser afin que j'invite le préfet [...] à mettre à votre disposition les livres qui auront été choisis $\|^{81}$. En janvier 1809, le préfet qui a transmis la liste de l'évêque, reçoit les instructions du ministre des Cultes : « S'il existe un exemplaire en double dans une bibliothèque ou un dépôt local, si l'exemplaire est inutile, il pourra être remis à M. l'évêque de Nancy ${ }^{82}$. Les bibliothèques de Bruyères, Darney, reçoivent de telles demandes de l'évêque et ne semblent guère y répondre.

En mai 1814, après le retour des Bourbon le nouveau préfet demande que 316 volumes soient sortis de la bibliothèque d'Épinal « pour former celle à l'usage particulier de M. le Préfet $\rangle^{83}$.

En pleine continuité des pratiques impériales, les opérations d'épuration des bibliothèques des livres en double, incomplets ou sans intérêt, se prolongent sous la Restauration et la monarchie de Juillet. Des milliers d'ouvrages sont ainsi vendus ou échangés. À Épinal, un «catalogue des

(80) Georges Baumont, « Notice historique de la bibliothèque municipale de Saint-Dié », op. cit., p. 250.

(81) AD Vosges, 6 T 1 B, lettre de l'évêque de Nancy, 19 décembre 1806.

(82) Ibid., lettre du ministre des Cultes au préfet des Vosges, 17 janvier 1809.

(83) BMI, AR2-226; catalogue des livres extraits... 18 mai 1814. 
livres doubles susceptibles d'être vendus » est dressé le $1^{\text {er }}$ mai 1820 . Il comprend essentiellement des ouvrages ecclésiastiques ${ }^{84}$. À Mirecourt, une liste est dressée en 1823, contenant deux colonnes, « livres à conserver, livres notables à vendre »; en avril 1835, un catalogue de 636 livres à vendre est dressé, une petite partie est vendue; une autre vente a lieu un peu plus tard, les acheteurs sont des ecclésiastiques et des hommes éclairés de la ville, et elle rapporte, pour les 452 livres destinés à être cédés, 2002 francs $^{85}$. C'est sous la monarchie censitaire que se développe une politique d'ouverture au public des bibliothèques avec la lecture sur place et des prêts soigneusement limités pour éviter des disparitions.

Finalement il n'y a pas eu de pérégrination des ouvrages et des bibliothèques tout au long de la Révolution, mais c'est la destination première qui a fondamentalement prévalu. Les livres rassemblés dans les chefs-lieux de district, Neufchâteau, Mirecourt, Remiremont ou SaintDié aussitôt après leur saisie, y sont finalement restés, malgré toutes les tentatives des administrations pour les regrouper au chef-lieu du département. La bibliothèque d'Épinal $\mathrm{a}$, comme les villes précitées, regroupé les bibliothèques des maisons religieuses de son district, dont Chaumousey, la plus importante, et a bénéficié de transferts importants sous l'Empire en provenance des grandes bibliothèques de la montagne.

Si les fonds saisis au début de la Révolution ont d'abord été conservés dans leur intégrité, c'est par la suite, sous l'Empire puis surtout au début du XIX ${ }^{\mathrm{e}}$ siècle qu'ils ont connu des déperditions considérables, dus aux mauvaises conditions de conservation, aux transferts vers d'autres bibliothèques (publiques ou ecclésiastiques), aux restitutions aux émigrés rentrés et surtout aux politiques de tri qui ont éliminé une multitude de livres jugés « inutiles », essentiellement des livres ecclésiastiques « théologiques ». S'il y a eu « vandalisme » dans les bibliothèques, celui-ci a été plutôt le fait des régimes post-révolutionnaires que de la Révolution triomphante! Que reste-t-il aujourd'hui de ces immenses bibliothèques? Quelques milliers d'ouvrages qui constituent le trésor de plusieurs bibliothèques publiques et qui sont bien protégés dans des réserves, ou, comme à Épinal, remarquablement mis en valeur dans des boiseries datant du $\mathrm{XVIII}{ }^{\mathrm{e}}$ siècle.

(84) Ibid., liasse d’Épinal.

(85) BM Mirecourt, dossier des sources de la bibliothèque. 
Les régimes qui ont dirigé la France pendant la Révolution et L'Empire, et les administrations départementales et municipales, ont su faire émerger la double mission de développement de la lecture publique et de la conservation du patrimoine écrit, double tâche qui continue de nos jours.

Jean Paul Rothiot

Université Nancy 2

88500 Frenelle-la-Grande

jp.cl.rothiot@orange.fr 